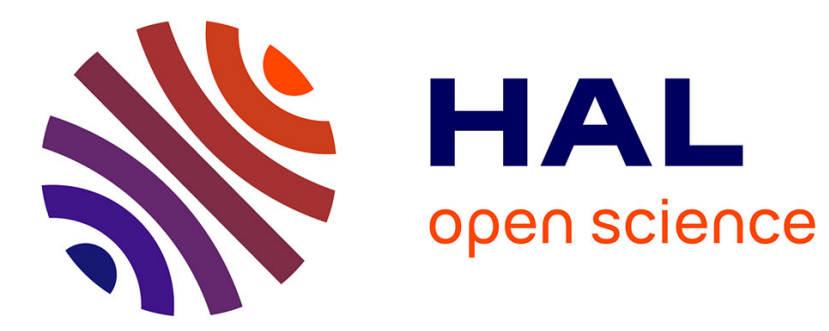

\title{
Perfectionnements du microphotomètre de chalonge
}

\author{
A. Ganson, M. Montel
}

\section{To cite this version:}

A. Ganson, M. Montel. Perfectionnements du microphotomètre de chalonge. J. Phys. Phys. Appl., 1958, 19 (S12), pp.171-175. 10.1051/jphysap:019580019012017100 . jpa-00212730

\section{HAL Id: jpa-00212730 https://hal.science/jpa-00212730}

Submitted on 1 Jan 1958

HAL is a multi-disciplinary open access archive for the deposit and dissemination of scientific research documents, whether they are published or not. The documents may come from teaching and research institutions in France or abroad, or from public or private research centers.
L'archive ouverte pluridisciplinaire HAL, est destinée au dépôt et à la diffusion de documents scientifiques de niveau recherche, publiés ou non, émanant des établissements d'enseignement et de recherche français ou étrangers, des laboratoires publics ou privés. 


\title{
PERFECTIONNEMENTS DU MICROPHOTOMETTRE DE CHALONGE
}

\author{
Par A. GANSON et M. MONTEL, \\ Laboratoire de Chimie Physique, Paris.
}

\begin{abstract}
Résumé. - On décrit un ensemble de modifications optiques et mécaniques du microphotomètre de Chalonge, qui permettent le dépouillement des spectres de grande hauteur enregistrés sur des plaques à forte granularité. Avec la nouvelle optique, on obtient, sur le cliché, des images de fentes de $25 \mathrm{~mm}$ de hauteur uniformément éclairées, Grâce à diverses adjonctions mécaniques, la fente-objet est réglable d'une manière continue et l'orientation relative des fentes et du cliché est plus précise. Un diaphragme supplémentaire permet d'opérer à flux constant sans modifier la température de couleur de la lampe. On a fait trois études sur des cas précis, afin d'apprécier le gain en reproductibilité et en résolution de l'appareil.
\end{abstract}

Abstract. - Optical and mechanical modifications are described, which have provided new improvements which allow the study of spectra of large size, recorded on large-grain plates. With the new optical system, slit images of $25 \mathrm{~mm}$ high and uniformly illuminated are given on the plate. With the help of several'new mechanical additions, the object-slit can be adjusted in a continuous way, and a better precision is obtained in the relative orientation of the slits and the plates. The addition of a new diaphragm allows operation with a constant flux without shifting the color temperature of the lamps. Three studies on particuliar cases have been made, in order to evaluate the effects of the improvements on the reproductibility and resolving power of the apparatus.

Le présent travail a été entrepris dans le but de rendre possible le dépouillement, à l'aide du microphotomètre Chalonge, de spectres de grande hauteur enregistrés sur des émulsions à forte granularité. Ce cas présente un intérêt particulier pour les études de spectroscopie $\mathrm{X}$ poursuivies dans notre laboratoire.

Dans l'appareil originel ( fig. 1a), on forme à l'aide d'un système optique A, l'image du filament d'une lampe de 6 watts $L$ dans un plan fixe $D_{1}$. Un jeu de fentes à lèvres fixes et de diaphragmes circulaires de dimensions variées que l'on dispose dans ce plan, permet d'obtenir une fente $F_{1}$ de dimensions choisies. Le plus grand diaphragme a $10 \mathrm{~mm}$ de diamètre. Le rayon moyen du faisceau lumineux sort horizontalement de $F_{1}$, est renvoyé verticalement par un miroir $M_{1}$ et traverse un miroir semi-argenté $M_{2}$ qui sert, pendant les réglages, à l'éclairage de la plaque photographique $\mathrm{P}$. A l'aide d'un condenseur mobile $\mathrm{O}$, on forme, de $\mathrm{F}_{1}$, sur le cliché à étudier $\mathrm{P}$, l'image $\mathrm{F}_{1}^{\prime}$ qui joue le rôle de fente d'exploration; sa hauteur maximum est $2,5 \mathrm{~mm}$. Le faisceau est repris par un objectif de position réglable $\mathrm{O}^{\prime}$; il est renvoyé horizontalement par un troisième miroir $\mathrm{M}_{3}$; l'image du cliché se forme sur une fente $\mathrm{F}_{2}$ placée devant une cellule photoélectrique C. Les montures du condenseur et de l'objectif, une fois retirées, laissent un passage libre de $30 \mathrm{~mm}$ de diamètre.

Les raies des spectres de rayons $\mathrm{X}$ obtenus à l'aide du spectrographe Cauchois ont une très faible courbure et une hauteur qui peut atteindre $6 \mathrm{~cm}$. La zone explorée par le spot n'est pas assez haute pour permettre, - dans le cas d'émulsions

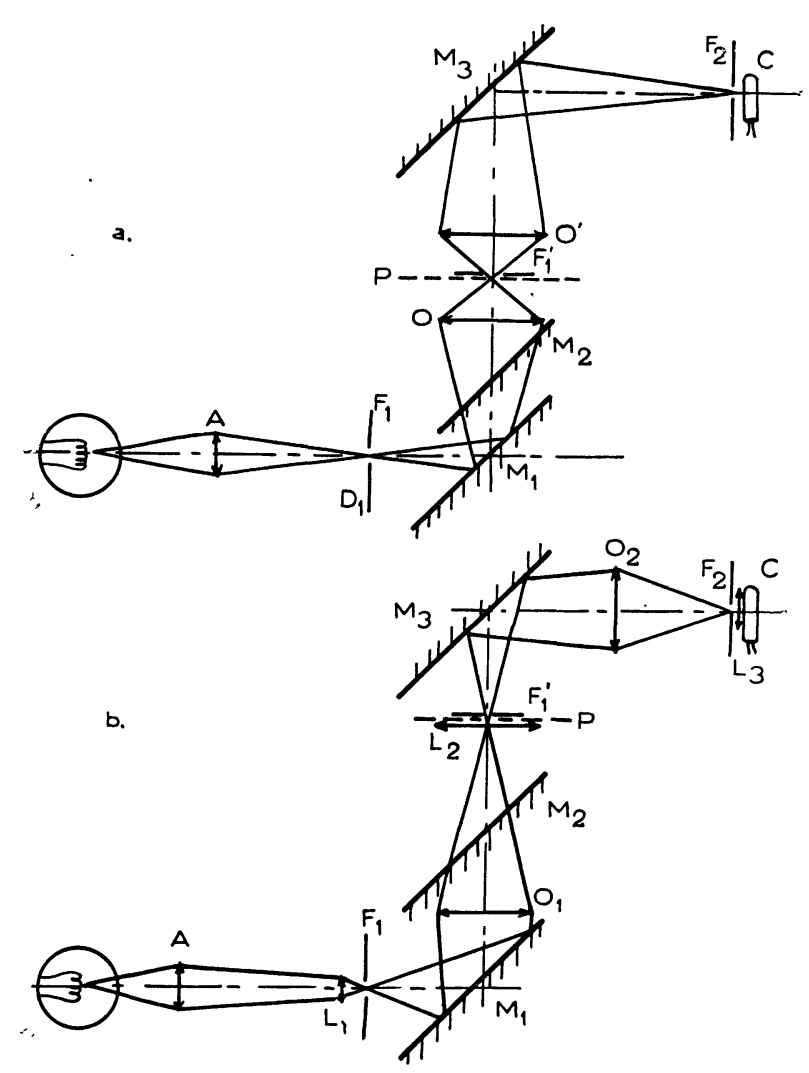

FIg. 1. - a) Montage de l'appareil originel. b) Montage modifié.

photographiques à forte granularité, - de distinguer les déviations du galvanomètre dues aux 
petits détails propres au spectre, de celles dues aux grains. Nous avons done été amenés à modifier l'optique, de manière à obtenir une fente d'exploration uniformément éclairée d'une hauteur aussi grande que possible. Cette modification en a entraîné d'autres, la précision nécessaire pour les différents réglages ayant de ce fait augmenté. La hauteur atteinte est théoriquement de $30 \mathrm{~mm}$, mais a dû être réduite, pour des impératifs mécaniques, à $25 \mathrm{~mm}$.

Changement de l'optique (fig. 1b). - Pour obtenir cette image de $25 \mathrm{~mm}$ de haut, il faut employer un condenseur donnant un grandissement de 2,5, donc situé à une distance de $F_{1}$ égale au 1/3,5 de la distance de la fente $F_{1}$ au cliché. Cette dernière est imposée par le montage et mesure $196 \mathrm{~mm}$ à quelques $\mathrm{mm}$ près. Le condenseur mentionné $\mathrm{O}_{1}$, doit par conséquent être placé entre les miroirs $M_{1}$ et $M_{2}$ et avoir une focale de l'ordre de $43 \mathrm{~mm}$. Pour des raisons de simplicité, nous avons pris l'objectif Nachet de focale $41 \mathrm{~mm}$ et de diamètre utile $8 \mathrm{~mm}$. Étant donné la place disponible, ce condenseur est fixe ; la fente doit donc être mobile pour permettre la mise au point sur le cliché. Sa position moyenne est à $59 \mathrm{~mm}$ de l'objectif, c'est-à-dire à $25 \mathrm{~mm}$ de l'ancienne fente.

Pour utiliser le maximum de la surface de la cellule $\mathrm{C}$, la fente de cellule $\mathrm{F}_{2}$ doit avoir sensiblement la même hauteur que la fente-objet. Un calcul analogue au précédent donne pour l'objectif $\mathrm{O}_{2}$ une focale de $53 \mathrm{~mm}$ environ ; nous avons pris l'objectif Nachet $55 \mathrm{~V}$. Cet objectif est monté après le troisième miroir $M_{3}$, avec mise au point par crémaillère.

Le montage terminé a montré : 1) que la diffusion par les objectifs est assez forte ; il y aurait avantage à utiliser des verres traités ; 2) que les fentes ne sont pas éclairées uniformément, par suite d'un effet d'œil de chat. Pour remédier à ces défauts, il a été nécessaire d'introduire trois lentilles de champ, de manière à conjuguer le doublet $\mathrm{A}$, le condenseur, l'objectif et la cellule, afin que le flux lumineux issu de l'un de ces éléments soit intégralement repris par l'élément suivant. Ces lentilles sont de simples verres de lunetterie. La première lentille $L_{1}$, située avant la fente-objet, a une focale de $43 \mathrm{~mm}$ et un diamètre de $15 \mathrm{~mm}$. La seconde lentille $\mathrm{L}_{2}$, placée avant le cliché, est fixée sur la monture de l'ancien condenseur C et amenée au contact de la plaque photographique ; elle a une focale de $50 \mathrm{~mm}$ et un diamètre de $29 \mathrm{~mm}$. La troisième lentille $L_{3}$, placée après la fente-image $F_{2}$ a une focale de $74 \mathrm{~mm}$ et un diamètre de $15 \mathrm{~mm}$. Il a fallu augmenter la longueur du miroir $\mathrm{M}_{3}$ pour ne pas diaphragmer le faisceau.

Les figures $1 a$ et $1 b$ montrent le montage avant et après transformation. L'opération de démon- tage d'une optique suivi du remontage de l'autre demande environ dix minutes.

Afin de ponvoir comparer les résultats obtenus avec les deux optiques, nous avons dépouillé un spectre X comprenant une discontinuité d'absorption faiblement contrastée, photographiée d'une part sur film à forte granularité et d'autre part sur plaque à grain fin ( $f$ ig. 2). Quelle que soit l'émulsion utilisée, les enregistrements $2 a$ et $2 c$, faits avec

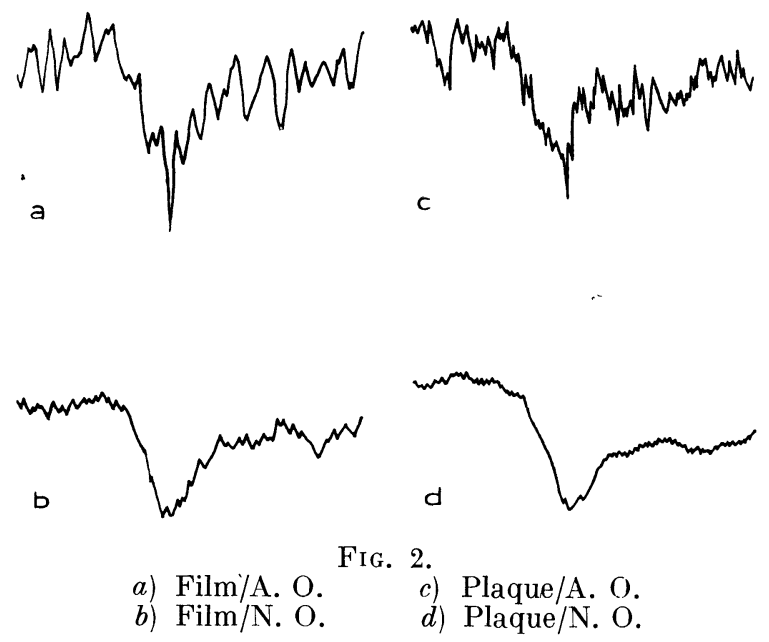

l'ancienne optique (A. O.) sont difficilement utilisables, les formes étant totalement masquées par la granularité. Les résultats obtenus avec la nouvelle optique (N. O.) (enregistrements $2 b$ et $2 d$ ) sont meilleurs, bien que la granularité du film reste encore trop forte pour que l'on puisse faire des mesures. Soulignons la structure très différente des émulsions de la plaque et du film. La première se compose de grains très petits uniformément répartis (d'où l'allure " hachée " du microphotogramme), tandis que la deuxième contient des " paquets" distincts de grosseur appréciable. L'amélioration obtenue est donc considérable : au lieu d'enregistrements inutilisables, l'appareil doit fournir dorénavant des microphotogrammes satisfaisants.

Modification du porte-plaque. - L'exploration simultanée d'une grande hauteur de la plaque photographique a souligné la nécessité de l'orienter avec une très grande précision. La vis d'orientation $X$ qui agit sur la perpendicularité des deux mouvements de translation du cliché est inutilisable pour le but cherché, car l'orthogonalité de ces deux mouvements doit être maintenue, étant une condition nécessaire pour que les enregistrements de deux dépouillements pris à des hauteurs différentes soient alignés. Il a fallu ajouter au porteplaque existant une platine tournante. De plus, nous avons modifié la vis $\mathrm{X}$ de façon que seule une clé spéciale permette de la manipuler, ce qui permet 
d'éviter un éventuel déréglage par inadvertance. La platine tournante est constituée par un cadre en duralumin percé d'une ouverture rectangulaire. Le cadre est monté sur deux portions d'un même cylindre, définissant un axe de rotation qui passe par le centre de l'ouverture rectangulaire. La rotation est assurée par une vis prenant appui sur le porte-plaque et contre une butée solidaire de la platine tournante. Un ressort de rappel assure le contact. A l'intérieur de l'ouverture rectangulaire pratiquée dans la platine glissent deux plaquettes parallèles, munies chacune de deux pattes métalliques, qui assurent la fixation du cliché et son immobilité relative pendant les enregistrements.

Reprenant les deux spectres utilisés plus haut, nous avons comparé les enregistrements obtenus en effectuant les réglages d'orientation des clichés d'abord à la main, puis à l'aide du nouveau dispositif. Les résultats semblent être meilleurs avec la platine tournante, mais il est évident qu'en opérant avec soin, les résultats devraient être identiques ; la seule différence réside dans la durée de l'opération ; elle peut se prolonger sans succès par tâtonnement en opérant à la main. Dans un cas d'espèce elle fut cinq fois plus longue que mécaniquement. Nous n'avons fait d'essais qu'avec l'ancienne optique, puisque les questions d'orientation relative ne dépendent pas du système utilisé. L'amélioration porte donc ici plus sur la commodité et la reproductibilité des réglages que sur la valeur des résultats.

Modification des fentes objet et image. - La fente-objet étant agrandie, les fentes interchangeables originelles (A. F.) ne donnent plus sur le cliché des images assez étroites pour permettre l'étude des fins détails. De plus, la monture de la fente présente du jeu, ce qui, - comme nous le verrons, - introduit une perte de résolution. Enfin, il était nécessaire, - comme nous l'avons dit, d'avancer la fente de $25 \mathrm{~mm}$ et de prévoir un dispositif de mise au point. Nous avons monté une fente dissymétrique (N. F.), dont la largeur est réglable à la valeur voulue. L'une des lèvres est fixée une fois pour toutes après les réglages préliminaires, de manière que son image coïncide avec la lèvre fixe de la fente de cellule. Une vis différentielle écartë les deux lèvres par pression sur la lèvre mobile (que deux ressorts tendent à appliquer contre la lèvre fixe). Ce système permet d'éviter l'écrasement des arètes. Le réglage en hauteur de la fente est assuré par un diaphragme-iris monté dans un support placé contre la fente. Son ouverture est commandé par le déplacement, le long d'une vis sans fin, d'un ergot fixé au levier du diaphragme.

Nous avons adjoint également un système précis d'orientation de la fente-objet. Il est constitué par une bague enserrant le cylindre portant la fente, le doublet A et la lampe et munie d'une tige carrée de plusieurs centimètres de longueur, dont l'extrémité se trouve serrée entre un système à pompe et l'extrémité d'une vis fixée au bâti.

Comme il n'y avait pas de système de centrage et d'orientation de la lampe, nous avons monté son culot entre trois vis calantes, l'orientation du filament se faisant à l'aide d'une bague de rotation. Nous avons remplacé la lampe de 6 watts par une lampe de 40 watts pour remédier à la perte de luminosité provoquée par l'augmentation de la longueur de la fente. Afin d'assurer un meilleur refroidissement, nous avons percé des trous dans la paroi latérale du cylindre.

Enfin, en vue d'avoir la même précision sur les fentes objet et de cellule, nous avons muni cette dernière d'une vis différentielle analogue à celle de la fente-objet et dix fois plus sensible que l'ancien système d'ouverture. Pour éviter des déréglages accidentels, nous avons supprimé l'extrémité facilement accessible de la vis d'orientation de cette fente et l'avons remplacée par une clé spéciale amovible.

Pour comparer l'ancien et le nouveau système de réglage des fentes, en utilisant successivement les deux optiques, nous avons fait quatre enregistrements (fig. 3). On constate que l'amélioration
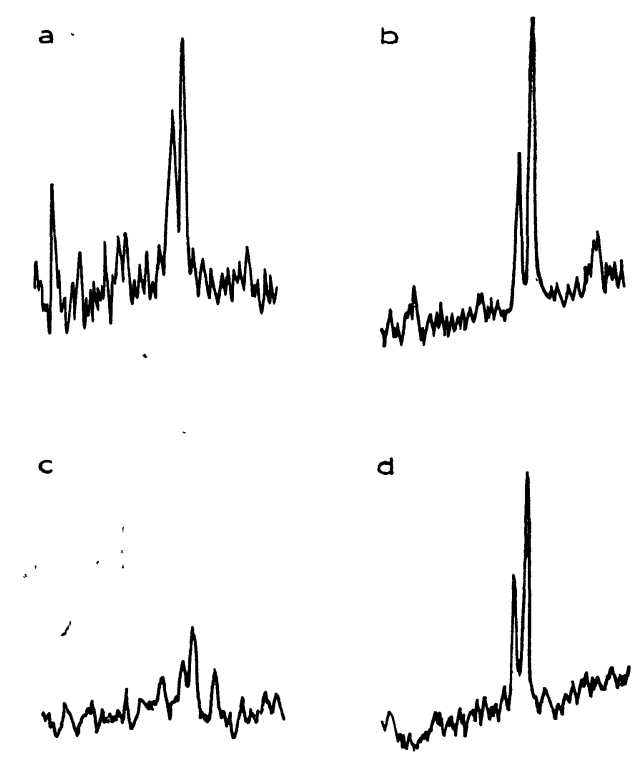

FIg. 3.
a) N. O./A. F.
c) A. O./A. F.

est plus grande avec l'ancienne optique. Cela provient sans doute du fait qu'il est plus facile d'orienter à la main une grande fente qu'une petite. Dans les deux cas, un défaut d'orientation de la fente entraîne une forte baisse de luminosité et de résolution. Ce nouveau montage est donc plus commode que l'ancien. De plus, l'orientation fixe de la fente 
permet un gain effectif sensible de la résolution de l'appareil.

Influence de la température de couleur (fig. 4).Dans le cadre de cet article nous ne nous sommes pas proposé une étude des performances photo-
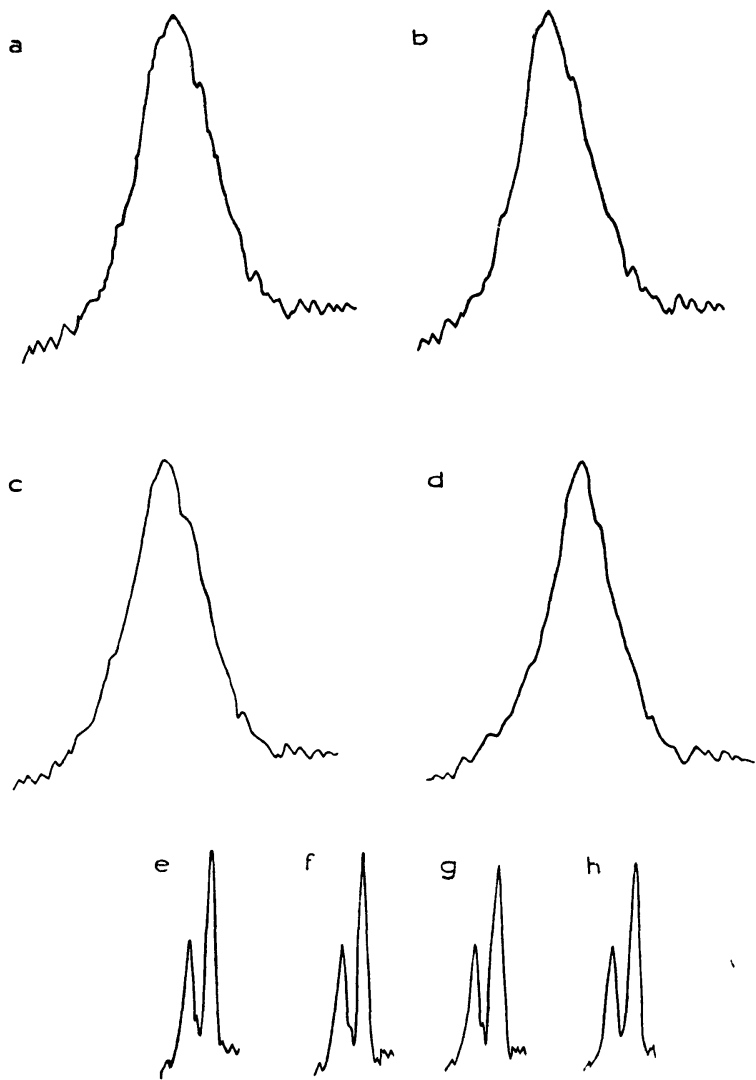

FIG. 4. - N.O. : a-d, plaque; $\mathrm{e}-\mathrm{h}$, film.

métriques de l'appareil. Les problèmes concernant la linéarité de la réponse et les mesures des densités seront examinés dans une prochaine étude. Toutefois, la modification mécanique décrite ci-dessous constitue une opération préliminaire nécessaire à l'étude de ces questions.

La diffusion et la transparence de la couche photographique sont étroitement liées à la composition du spectre de la lumière qui tombe sur elle. Jusqu'ici les variations de la sensibilité du microphotomètre se faisaient en agissant sur l'intensité du courant de la lampe, donc sur la température de couleur, ce qui peut fausser complètement les résultats de la photométrie quantitative. Afin d'opérer à débit constant, - ce qui permet d'ailleurs un fonctionnement plus homogène des accumulateurs, - nous avons muni l'objectif $\mathrm{O}_{1}$ d'un diaphragme-iris permettant de réduire le flux lumineux. Une tige articulée, d'une dizaine de centimètres de long, règle son ouverture.
Nous avons donné à la lampe quatre intensités différentes, tout en fermant le diaphragme jusqu'à l'obtention de la même déviation du spot d'enregistrement, et nous avons fait chaque fois un enregistrement avec la plaque et avec le film. Si nous traçons la courbe de l'intensité I du courant dans la cellule en fonction de l'intensité i du courant dans la lampe, on voit que nous opérions à $f I \mathrm{~d} i$ constant.

L'augmentation de la diffusion avec le débit de la lampe est très nette, surtout dans le cas de la plaque. La largeur et la forme des raies changent, d'où nécessité en photométrie d'avoir un flux toujours identique. Nous n'avons pas fait d'essai avec l'ancienne optique, car les objectifs fournis sont dépourvus de diaphragme, et le montage d'un diaphragme provisoire serait compliqué. Par conséquent, le nouveau montage permet d'étudier des plaques de densités optiques variées avec la même intensité électrique dans la lampe et une température de couleur bien définie.

\section{Études complémentaires}

Nous avons complété notre étude pratique par un examen rapide des problèmes suivants, que nous ne décrirons que brièvement.

a) Influence de la granularité sur la réponse du microphotomètre. - La comparaison entre les courbes représentées sur la figure 3 est éloquente. On peut dire approximativement que les formes obtenues n'ont un sens que si la granularité ne provoque pas autour de la courbe moyenne des déviations supérieures à $1 / n$ de celles dûes au phénomène étudié ; $n=10$ semble être, d'après l'examen des enregistrements, une base raisonnable. Toutes les plaques de granularité relative supérieure doivent être éliminées.

b) Influence de la vitesse d'entraînement du cliché. - Pour une plaque donnée, le grandissement et la vitesse de translation du chariot porte-papier doivent être tels que l'inertie totale de l'appareil n'intervienne pas. A titre d'exemple, les distances entre les sommets des deux raies d'un doublet et le minimum qui les sépare étaient de 9 et $16 \mathrm{~mm}$; aux grandes vitesses et pour de petits grandissements elles ont été ramenées à 2 et $12 \mathrm{~mm}$. Cela revient à dire que les mesures que l'on effectuait sur l'enregistrement n'avait plus aucun sens et que les formes étaient grandement affectées. Il importe donc de choisir chaque fois la vitesse d'entraînement du cliché de manière que la vitesse de rotation du spot d'enregistrement ne soit pas influencée par l'inertie du galvanomètre.

De plus, nous avions remarqué une oscillation parasite du spot dûe à un mauvais guidage du cliché. Une notable amélioration de ce guidage a été 
obtenue grâce au remplacement de la portée existante, faite de trois roulements à bille, par deux V et un plat.

c) Influence de la lumière diffuse ( $f i g .5$ ), - Cette lumière vient des réflexions parasites sur les différentes pièces optiques et mécaniques et de la dif-

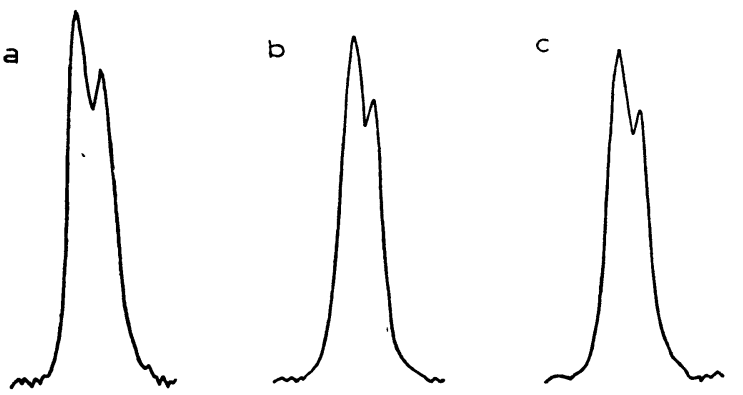

Fig. 5. - Doublet de référence. - N. O.

a) Sans lumière parasite.

b) Lumière parasite transmise.

c) Lumière parasite réfléchie.

fusion dûe au cliché ; elle limite le seuil de contraste. Nous avons fait un essai en juxtaposant au faisceau laminaire normal un éclairage de champ traversant le cliché ; ensuite nous lui avons ajouté la lumière ambiante qui se réfléchit sur le cliché. Ceci permit d'observer l'influence de la diffusion de la gélatine en lumière transmise et en lumière réfléchie. Les résultats sont conformes aux prévisions théoriques, à savoir, que le contraste a baissé, c'est-à-dire que, par exemple, l'écart entre la base d'une raie et son sommet est plus faible. Il y aurait donc intérêt à reprendre l'appareil pour " vernir mat " les pièces mécaniques et " traiter " les surfaces optiques.

Il nous restait à étudier dans les meilleurs conditions possibles les performances de l'appareil et à les comparer si possible, aux résultats donnés par d'autres appareils. A cette fin, nous avons étudié, en qualité de test, le spectre d'un doublet $\mathrm{K} \alpha$ particulièrement fin, obtenu sur une plaque à grain fin.

d) Performances de l'instrument. - Nous avons d'abord dégrossi les réglages au grandissement 7, en retouchant l'orientation de la plaque photographique jusqu'à séparer le doublet mentionné. Ce grandissement convient bien, car un léger déplacement du doublet ne le fait pas sortir du champ. Nous avons ensuite achevé la mise au point au grandissement 50, les réglages étant plus précis, puisque la plaque se déplace moins rapidement. Nous avons pu ainsi séparer deux raies fines distantes de $90 \mu$ sur le cliché, les déviations du spot d'enregistrement mesurées sur les enregistrements étant respectivement (par rapport au fond continu) de 0,88 et 0,73 pour la raie la plus faible et pour le minimum entre les raies du doublet, la réponse de la raie la plus intense étant prise pour unité (fig. 6). Ce résultat constitue un gain impor-

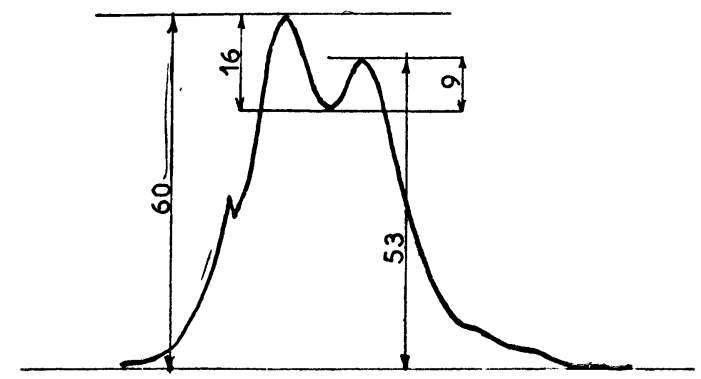

FIG. 6.

tant sur les résultats antérieurs obtenus avec l'ancienne optique, qui ne permettait pas la résolution de ce doublet. Toutefois, la comparaison avec un enregistrement fait sur un microphotomètre Kipp modifié a montré qu'il était possible d'obtenir mieux. La différence semble être liée d'une part à un mauvais guidage en translation de la plaque, et d'autre part à l'existence d'une forte lumière parasite dans l'appareil, lumière qu'il ne serait possible d'atténuer qu'en effectuant les améliorations envisagées et en supprimant les miroirs semi-argentés.

Quoi qu'il en soit, cet appareil nous fournit à présent des enregistrements microphotométriques tout à fait satisfaisants de spectres de rayons $\mathrm{X}$ enregistrés sur plaques à forte granularité. Les facultés d'emploi ont été augmentées et les performances de l'appareil sensiblement accrues.

Les modifications mécaniques et optiques ont été effectuées par M. Richard au Laboratoire de Chimie-Physique de la Faculté des Sciences de Paris, à l'exception du montage des objectifs Nachet, qui a été exécuté par M. Angelé dans son atelier.

Nous tenons à exprimer ici votre vive reconnaissance à $M$. Chalonge, qui nous a encouragé dans notre travail et a procédé aux premiers essais de l'appareil.

Manuscrit reçu le 15 octobre 1958. 